\title{
Effect of some medicinal plants on liver and kidney functions in diabetic albino rats
}

\author{
Eman G. E. Helal"' Ashraf Mahmoud **, Mervat Abdel-Rahman ***, \\ and Anwaar A. Kahwash* \\ * Zoology Department, Faculty of Science, Al-Azher University (Girls) \\ ** Histology Department, Faculty of Medicine, Al-Azher University \\ ***Clinical Pathology Lab., Student Hospital, Cairo University, Giza.
}

\begin{abstract}
The present study aimed to clarify the effect of aqueous extracts of five plants (Nigella sativa, Aloe vera, Ferula assafoetida, Boswellia carterii Birdw and Commiphora myrrha) and their mixture on liver and kidney functions and protein profiles. Eighty adult male albino rats were divided into eight groups, the first served as control group, other groups were injected with alloxan $(120 \mathrm{mg} / \mathrm{kg} \mathrm{b}$.wt). The second group served as diabetic rats, the third were treated with a mixture $(0.1 \mathrm{~g} / 100 \mathrm{~g}$ b.wt $)$, the fourth was treated with Nigella sativa $(0.1 \mathrm{~g} / 100 \mathrm{~g} \mathrm{~b} . \mathrm{wt})$, the fifth was treated with Aloe vera $(0.05 \mathrm{~g} / 100 \mathrm{~g}$ b.wt $)$, the sixth was treated with Ferula assafoetida $(0.1 \mathrm{~g} / 100 \mathrm{~g}$ b.wt $)$, the seventh was treated with Boswellia carterri Birdw $(0.1 \mathrm{~g} / 100 \mathrm{~g}$ b.wt $)$ and the eighth group was treated with Commiphora myrrha $(0.05 \mathrm{~g} / 100 \mathrm{~g} \mathrm{~b} . w \mathrm{t})$. After thirty days of treatment half of each group was decapitated and the other one was left for 15 days without any additional treatment as recovery period to followup their hazards if present.

The results revealed highly significant increase $(\mathrm{p}<0.01)$ in serum transaminases (aspartate ( AST ) and alanine (ALT)), alkaline phosphates (ALP), total bilirubin, urea and creatinine and recorded highly significant decrease $(\mathrm{p}<0.01)$ in serum total protein, albumin and globulin concentrations in the diabetic group when compared with normal rates. Otherwise, all plants extracts treated groups showed insignificant changes in the previous parameters when compared with control one .

It seams, therefore that the water extracts of these plants and their mixture have protective effect against the side effects of alloxan on liver and kidney.
\end{abstract}

Key words: Liver functions, kidney functions, diabetic Albino rats.

\section{Introduction}

One of the most important clinical features of diabetes is its association with chronic tissue complications (Wiliams and Pick. Up, 2000). Liver tissue one of these tissues damaged by diabetes causing cell necrosis (Boorman et al.,1990).

About 170 phytoconstituents isolated from 110 plants belonging to 55 families were stated to possess liver-protective activity; about 600 commercial herbal formulations with claimed hepatoprotictive activity are being marketed world-wide. Otherwise, some plant materials (such as those containing pyrrolizidine alkaloids) considered one of causative factors of liver disorders (Evans, 2001).

The present study was a trial to clarify if the mixture of Nigella sativa, Aloe vera, Ferula assafoetida, Boswellia carterii Birdw and Commiphora myrrha and each plant alone have liver-protective activity or causative factors of liver disorders. And their effect on kidney function.

\section{Material and Methods}

Eighty mature male albino rats (weight $120 \pm 20 \mathrm{gm}$ ). Rats divided into 
two groups, the first group (10 rats) served as control and the second group (70 rats) were fasted over night, then injected with a single subcutaneous dose of alloxan $(120 \mathrm{mg} / \mathrm{kg} \mathrm{b}$. wt.). After 48 hours of alloxan injection, blood glucose level measured by glucometer. Rats with fasting blood glucose level more than $250 \mathrm{mg} / \mathrm{dl}$ considered diabetic, they divided into seven subgroups each containing 10 rats in separate cages as the following:

- Diabetic group.

- Mixture treated group $(0.1 \mathrm{~g} /$ $100 \mathrm{~g}$ b.wt).

- Nigella sativa treated group (0.1g/ 100g b.wt).

- Aloe vera treated group $(0.05 \mathrm{~g} /$ $100 \mathrm{~g}$ b.wt).

- Ferula assafoetida treated group (0.1g/ 100g b.wt).

- Boswellia carterii Birdw treated group (0.1g/ 100g b.wt).

- Commiphora myrrha treated group $(0.05 \mathrm{~g} / 100 \mathrm{~g} \mathrm{b.wt})$

\section{Preparations of plants water extractions:}

\section{A - The mixture:}

Plants were grinned and $10 \mathrm{gm}$ of each plant were mixed and boiled in $100 \mathrm{ml}$ dist. water for a period of $10 \mathrm{~min}$ then cooled to room temperature and filtered. The extract was given orally with a dose of $1 \mathrm{ml} / 100 \mathrm{gm}$ $b$. wt. (every day till 30 days).

\section{B-Nigella sativa:}

The aqueous extract of $N$. sativa was prepared by boiling $50 \mathrm{gm}$ of the plant with $200 \mathrm{ml}$ dist water for $10 \mathrm{~min}$ (note: when $50 \mathrm{gm}$ of the plant boiled with $100 \mathrm{ml}$ dist. water there was no extract because the plant absorbed all water). After cooling to room temperature, the extract was filtered and stored in refrigerator. The dose used was $1 \mathrm{ml} / 100 \mathrm{~b}$. wt. daily as oral dosage for 30 successive days.

C- Commiphora myrrha, Boswellia Carterii Birdw, Aloe vera, and Ferula assa-foetida:

Their aqueous extract was prepared by boiling $50 \mathrm{gm}$ of each plant alone in $100 \mathrm{ml}$ dist. water for $10 \mathrm{~min}$. After cooling to room temperature, the extract was filtered and stored in refrigerator. The dose used was $1 \mathrm{ml} / 100 \mathrm{~g} b$. wt. daily as oral dosage for 30 days. Except Aloe vera and Commiphora myrrha treated animals, where the dose used was $1 / 2 \mathrm{ml} / 100 \mathrm{~g} \quad b$. wt. daily as oral dosage for 30 days $(1 \mathrm{ml} / 100 \mathrm{~g} b$. wt. cause death of some rats, so, we used the half dose).

After 30 days of treatment, 5 animals of each group were decapitated, while the other half of each group was kept for 15 days more, without any additional treatment to follow up if there is any delayed effect of the treatment.

Blood sera were collected for the determination of aspartate transaminase (AST) and alanine transaminase (ALT) (Reitman and Frankel, 1975), lactatdehydra ( LDH ) (Kachmar and Moss, 1976), alkaline phosphatase (John, 1982), total bilirubin (Tietz, 1986), urea (Patton and Crouh, 1977), creatinine (Jeffe, 1886), total protein (Doumas, 1975) and albumin (Webster, 1977). Significant differences between the means of control and treated groups were considered at $\mathrm{p}<0.05$ (Sokal and Rohif, 1981).

\section{Results}

The current study indicated high significant increase $\quad(p<0.01)$ in serum aspartate transaminase (AST), alanine transaminase (ALT) and alkaline phosphatase in alloxan treated group when compared with control group during the experimental period (Tables 1a,b,2a,b \& 3a,b).

All plant extracts groups showed insignificant change in AST, ALT, alkaline phosphatase and LDH activities when compared with control rats except Ferule assa-foetida treated group which showed a significant increase $(p<0.05)$ after treatment and recovery periods. On the other hand, highly significant decrease $(\mathrm{p}<0.01)$ was recorded in plant extracts treated groups when compared with diabetic group throughout the experiment (Tables 1a,b, $2 a, b$ \& 3a,b).

Concerning total bilirubin concentration, it was highly significant increased $(p<0.01)$ in diabetic group when compared with control rats during the experimental period (Tables 4a,b) . 
All plant extracts treated groups showed no significant change in total bilirubin concentration when compared with control group, while highly significant decrease $(p<0.01)$ was recorded in comparison with diabetic group throughout the experiment (Tables $4 a, b)$.

The current study showed high significant increase $(p<0.01)$ in serum urea and creatinine concentration in diabetic group when compared with control one throughout the experiment (Tables 5a,b \& 6a,b).

On the other hand, all plant extracts treated groups showed no significant change in the previous two parameters when compared with control group. In the same time highly significant decrease $(\mathrm{p}<$ 0.01) was observed when compared with diabetic group during the experimental period (Tables 5a,b \& 6a,b).

Table (1 - A) :-Aspartate transaminase ( AST ) activity in control, diabetic and plant

\begin{tabular}{|c|c|c|c|c|c|c|c|c|c|}
\hline \multirow{2}{*}{\multicolumn{2}{|c|}{ parameter }} & \multicolumn{8}{|c|}{ Treated period ( 4 weeks ) } \\
\hline & & Control & Diabetic & Mixture & Nigella & Aloe & $\begin{array}{c}\text { Ferula } \\
\text { assa- }\end{array}$ & $\begin{array}{c}\text { Boswellia } \\
\text { carterii }\end{array}$ & $\begin{array}{c}\text { Commi- } \\
\text { phora }\end{array}$ \\
\hline \multirow{4}{*}{ 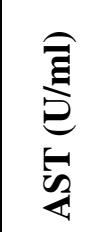 } & Mean & 38.50 & 51.80 & 33.00 & 37.90 & 43.00 & 45.90 & 37.00 & 37.01 \\
\hline & $\pm \mathrm{SE}$ & 1.84 & 1.72 & 1.94 & 1.68 & 1.50 & 2.15 & 1.84 & 1.27 \\
\hline & $\mathbf{A}$ & & $* *$ & & & & $*$ & & \\
\hline & B & & & $* *$ & $* *$ & $* *$ & $* *$ & $* *$ & $* *$ \\
\hline
\end{tabular}

extracts trated rats after 4 weeks of treatment .

Table (1-B) :-Aspartate transaminase ( AST) activity in control, diabetic and plant extracts trated rats after 2 weeks of recovery period.

\begin{tabular}{|c|c|c|c|c|c|c|c|c|c|}
\hline & & \multicolumn{8}{|c|}{ Recovery period ( 2 weeks ) } \\
\hline & & Control & Diabetic & Mixture & $\begin{array}{c}\text { Nigella } \\
\text { sativa }\end{array}$ & $\begin{array}{l}\text { Aloe } \\
\text { vera }\end{array}$ & $\begin{array}{c}\text { Ferula } \\
\text { assa- } \\
\text { foetida }\end{array}$ & $\begin{array}{c}\text { Boswellia } \\
\text { carterii } \\
\text { Birdw }\end{array}$ & $\begin{array}{c}\text { Commi- } \\
\text { phora } \\
\text { myrrha }\end{array}$ \\
\hline \multirow{4}{*}{ 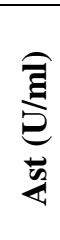 } & Mean & 30.6 & 60.2 & 34.7 & 30.2 & 30.3 & 36.91 & 30.2 & 31.7 \\
\hline & $\pm \mathbf{S E}$ & 1.713 & 1.715 & 1.068 & 1.554 & 1.545 & 2.023 & 1.554 & 1.375 \\
\hline & $\mathbf{A}$ & & $* *$ & & & & $*$ & & \\
\hline & B & & & $* *$ & $* *$ & ** & ** & $* *$ & $* *$ \\
\hline
\end{tabular}

A - in comparison with control group .

$\mathrm{B}$-in comparison with diabetic group .

$* \mathrm{p}<0.05$. 
$* * p<0.01$.

Table (2-A): Alanine transaminase ( ALT) activity in control, diabetic and plant extracts treated rats after 4 weeks of treatment .

\begin{tabular}{|c|c|c|c|c|c|c|c|c|c|}
\hline & \multicolumn{8}{|c|}{ Treated period ( 4 weeks ) } \\
\hline & & Control & Diabetic & Mixture & $\begin{array}{l}\text { Nigella } \\
\text { sativa }\end{array}$ & $\begin{array}{l}\text { Aloe } \\
\text { vera }\end{array}$ & $\begin{array}{c}\text { Ferula } \\
\text { assa- } \\
\text { foetida }\end{array}$ & $\begin{array}{c}\text { Boswellia } \\
\text { carterii } \\
\text { Birdw }\end{array}$ & $\begin{array}{c}\text { Commi- } \\
\text { phora } \\
\text { myrrha }\end{array}$ \\
\hline \multirow{4}{*}{ 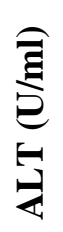 } & Mean & 21.20 & 33.90 & 20.40 & 19.80 & 23.40 & 26.60 & 22.40 & 20.60 \\
\hline & $\pm \mathrm{SE}$ & 1.43 & 0.86 & 1.89 & 1.49 & 1.29 & 0.98 & 1.47 & 0.98 \\
\hline & $\mathbf{A}$ & & ** & & & & * & & \\
\hline & B & & & $\star \star$ & $\star *$ & $\star \star$ & ** & $\star \star$ & ** \\
\hline
\end{tabular}

Table (2-B) : Alanine transaminase ( ALT) activity in control, diabetic and plant extracts treated rats after 2 weeks of recovery period.

\begin{tabular}{|c|c|c|c|c|c|c|c|c|c|}
\hline & \multicolumn{8}{|c|}{ Recovery period ( 2 weeks ) } \\
\hline & & Control & Diabetic & Mixture & $\begin{array}{l}\text { Nigella } \\
\text { sativa }\end{array}$ & $\begin{array}{l}\text { Aloe } \\
\text { vera }\end{array}$ & $\begin{array}{c}\text { Ferula } \\
\text { assa- } \\
\text { foetida }\end{array}$ & $\begin{array}{c}\text { Boswellia } \\
\text { carterii } \\
\text { Birdw }\end{array}$ & $\begin{array}{c}\text { Commi- } \\
\text { phora } \\
\text { myrrha }\end{array}$ \\
\hline \multirow{4}{*}{ 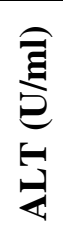 } & Mean & 22.00 & 31.80 & 21.20 & 17.40 & 24.00 & 27.60 & 21.40 & 24.60 \\
\hline & $\pm \mathrm{SE}$ & 1.80 & 1.05 & 3.64 & 2.56 & 1.80 & 1.36 & 0.96 & 1.86 \\
\hline & $\mathbf{A}$ & & $* *$ & & & & $*$ & & \\
\hline & B & & & $* *$ & $* *$ & $* *$ & $* *$ & $* *$ & $* *$ \\
\hline
\end{tabular}

A -in comparison with control group .

$\mathrm{B}$-in comparison with diabetic group

$* \mathrm{p}<0.05$.

$* * \mathrm{p}<0.01$.

Table (3-A) : Alkaline phosphatase activity in control, diabetic and plant extracts treated rats after 4 weeks of treated period .

\begin{tabular}{|c|c|c|c|c|c|c|c|c|c|}
\hline & & \multicolumn{8}{|c|}{ Treated period ( 4 weeks ) } \\
\hline & & Control & Diabetic & Mixture & $\begin{array}{l}\text { Nigella } \\
\text { sativa }\end{array}$ & $\begin{array}{l}\text { Aloe } \\
\text { vera }\end{array}$ & $\begin{array}{c}\text { Ferula } \\
\text { assa- } \\
\text { foetida }\end{array}$ & $\begin{array}{c}\text { Boswellia } \\
\text { carterii } \\
\text { Birdw }\end{array}$ & $\begin{array}{c}\text { Commi- } \\
\text { phora } \\
\text { myrrha }\end{array}$ \\
\hline \multirow{4}{*}{ 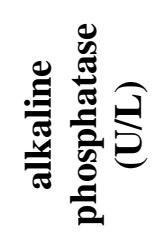 } & Mean & 91.38 & 116.84 & 91.64 & 92.98 & 92.30 & 94.30 & 90.32 & 90.92 \\
\hline & $\pm \mathbf{S E}$ & 0.33 & 0.45 & 0.40 & 0.96 & 0.51 & 1.08 & 0.83 & 0.74 \\
\hline & $\mathbf{A}$ & & ** & & & & * & & \\
\hline & B & & & ** & $\star *$ & ** & $* *$ & $\star *$ & $\star *$ \\
\hline
\end{tabular}


Table(3-B) : Alkaline phosphatase activity in control, diabetic and plant extracts treated rats after 2 weeks of recovery period .

\begin{tabular}{|c|c|c|c|c|c|c|c|c|c|}
\hline & & \multicolumn{8}{|c|}{ Recovery period ( 2 weeks ) } \\
\hline & & Control & Diabetic & Mixture & $\begin{array}{c}\text { Nigella } \\
\text { sativa }\end{array}$ & $\begin{array}{l}\text { Aloe } \\
\text { vera }\end{array}$ & $\begin{array}{c}\text { Ferula } \\
\text { assa- } \\
\text { foetida }\end{array}$ & $\begin{array}{c}\text { Boswellia } \\
\text { carterii } \\
\text { Birdw }\end{array}$ & $\begin{array}{c}\text { Commi- } \\
\text { phora } \\
\text { myrrha }\end{array}$ \\
\hline \multirow{4}{*}{ 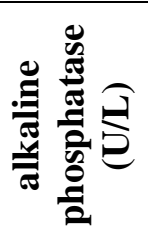 } & Mean & 91.02 & 114.74 & 91.10 & 92.56 & 92.48 & 94.58 & 91.80 & 89.80 \\
\hline & $\pm \mathbf{S E}$ & 0.80 & 0.88 & 0.59 & 0.70 & 0.44 & 0.78 & 0.77 & 0.48 \\
\hline & $\mathbf{A}$ & & $\star \star$ & & & & * & & \\
\hline & B & & & ** & ** & ** & ** & ** & ** \\
\hline
\end{tabular}
A - in comparison with control group .
$\mathrm{B}$ - in comparison withdiabetic group .
$* \mathrm{p}<0.05$.
${ }^{* *} p<0.01$

Table (4 -A) :Total bilirubin concentration in control, diabetic and plant extracts treated rats after 4 weeks of treated period.

\begin{tabular}{|c|c|c|c|c|c|c|c|c|c|}
\hline & & \multicolumn{8}{|c|}{ Treated period ( 4 weeks ) } \\
\hline & & Control & Diabetic & Mixture & $\begin{array}{c}\text { Nigella } \\
\text { sativa }\end{array}$ & $\begin{array}{l}\text { Aloe } \\
\text { vera }\end{array}$ & $\begin{array}{l}\text { Ferula } \\
\text { assa- } \\
\text { foetida }\end{array}$ & $\begin{array}{c}\text { Boswellia } \\
\text { carterii } \\
\text { Birdw }\end{array}$ & $\begin{array}{c}\text { Commi- } \\
\text { phora } \\
\text { myrrha }\end{array}$ \\
\hline \multirow{4}{*}{ 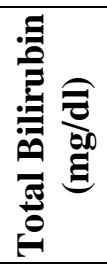 } & Mean & 0.90 & 1.96 & 0.82 & 0.88 & 0.86 & 1.08 & 0.68 & 0.73 \\
\hline & $\pm \mathbf{S E}$ & 0.10 & 0.09 & 0.18 & 0.19 & 0.08 & 0.05 & 0.02 & 0.04 \\
\hline & $\mathbf{A}$ & & $\star \star$ & & & & & & \\
\hline & B & & & ** & ** & ** & $\star \star$ & ** & ** \\
\hline
\end{tabular}

Table (4-B) : Total bilirubin concentration in control, diabetic and plant extracts treated rats after 2 weeks of recovery period .

\begin{tabular}{|c|c|c|c|c|c|c|c|c|c|}
\hline & \multicolumn{8}{|c|}{ Recovery period ( 2 weeks ) } \\
\hline & & Control & Diabetic & Mixture & $\begin{array}{c}\text { Nigella } \\
\text { sativa }\end{array}$ & $\begin{array}{l}\text { Aloe } \\
\text { vera }\end{array}$ & $\begin{array}{c}\text { Ferula } \\
\text { assa- } \\
\text { foetida }\end{array}$ & $\begin{array}{c}\text { Boswellia } \\
\text { carterii } \\
\text { Birdw }\end{array}$ & $\begin{array}{c}\text { Commi- } \\
\text { phora } \\
\text { myrrha }\end{array}$ \\
\hline \multirow{4}{*}{ 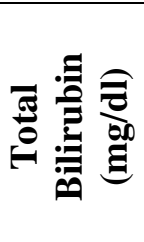 } & Mean & 1.06 & 1.94 & 0.88 & 0.69 & 1.09 & 1.04 & 0.83 & 0.82 \\
\hline & $\pm \mathbf{S E}$ & 0.15 & 0.09 & 0.18 & 0.11 & 0.01 & 0.08 & 0.03 & 0.04 \\
\hline & $\mathbf{A}$ & & ** & & & & & & \\
\hline & B & & & ** & ** & ** & ** & ** & ** \\
\hline
\end{tabular}

A - in comparison with control group .

$\mathrm{B}$ - in comparison with diabetic group .

$\mathrm{p}<0.05$.

$* * p<0.01$ 
Table (5-A) : Serum urea level in control, diabetic and plant extracts treated rats after 4 weeks of tretment .

\begin{tabular}{|c|c|c|c|c|c|c|c|c|c|}
\hline & & \multicolumn{8}{|c|}{ Treated period ( 4 weeks ) } \\
\hline & & Control & Diabetic & Mixture & $\begin{array}{c}\text { Nigella } \\
\text { sativa }\end{array}$ & $\begin{array}{l}\text { Aloe } \\
\text { vera }\end{array}$ & $\begin{array}{c}\text { Ferula } \\
\text { assa- } \\
\text { foetida }\end{array}$ & $\begin{array}{c}\text { Boswellia } \\
\text { carterii } \\
\text { Birdw }\end{array}$ & $\begin{array}{c}\text { Commi- } \\
\text { phora } \\
\text { myrrha }\end{array}$ \\
\hline \multirow{4}{*}{ 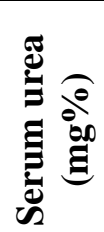 } & Mean & 36.83 & 89.33 & 32.76 & 33.14 & 38.60 & 36.15 & 34.50 & 32.92 \\
\hline & $\pm \mathrm{SE}$ & 1.64 & 1.25 & 1.51 & 1.21 & 0.78 & 0.68 & 1.32 & 0.79 \\
\hline & $\mathbf{A}$ & & ** & & & & & & \\
\hline & B & & & ** & ** & ** & ** & ** & ** \\
\hline
\end{tabular}

Table (5-B) : Serum urea level in control, diabetic and plant extracts treated rats after 2 weeks of recovery period .

\begin{tabular}{|c|c|c|c|c|c|c|c|c|c|}
\hline & \multicolumn{8}{|c|}{ Recovery period ( 2 weeks ) } \\
\hline & & Control & Diabetic & Mixture & $\begin{array}{l}\text { Nigella } \\
\text { sativa }\end{array}$ & $\begin{array}{l}\text { Aloe } \\
\text { vera }\end{array}$ & $\begin{array}{c}\text { Ferula } \\
\text { assa- } \\
\text { foetida }\end{array}$ & $\begin{array}{c}\text { Boswellia } \\
\text { carterii } \\
\text { Birdw }\end{array}$ & $\begin{array}{c}\text { Commi- } \\
\text { phora } \\
\text { myrrha }\end{array}$ \\
\hline \multirow{4}{*}{ 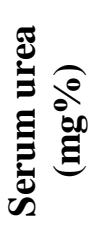 } & Mean & 33.25 & 79.63 & 31.84 & 32.83 & 33.32 & 29.84 & 35.23 & 31.38 \\
\hline & $\pm \mathrm{SE}$ & 1.31 & 0.81 & 1.58 & 0.77 & 1.00 & 1.02 & 1.22 & 0.79 \\
\hline & $\mathbf{A}$ & & ** & & & & & & \\
\hline & B & & & ** & ** & ** & ** & ** & ** \\
\hline
\end{tabular}

A - in comparison with control group .

$\mathrm{B}$ - in comparison with diabetic group .

$\mathrm{p}<0.05$.

$* * \mathrm{p}<0.01$.

Table ( 6 - A ) : Serum creatinine level in control, diabitic and plant extracts treated rats after 4 weeks of treated period

\begin{tabular}{|c|c|c|c|c|c|c|c|c|c|}
\hline & \multicolumn{8}{|c|}{ Treated period ( 4 weeks) } \\
\hline & & Control & Diabetic & Mixture & $\begin{array}{c}\text { Nigella } \\
\text { sativa }\end{array}$ & $\begin{array}{l}\text { Aloe } \\
\text { vera }\end{array}$ & $\begin{array}{c}\text { Ferula } \\
\text { assa- } \\
\text { foetida }\end{array}$ & $\begin{array}{c}\text { Boswellia } \\
\text { carterii } \\
\text { Birdw }\end{array}$ & $\begin{array}{c}\text { Commi- } \\
\text { phora } \\
\text { myrrha }\end{array}$ \\
\hline \multirow{4}{*}{ 童言 } & Mean & 0.91 & 1.47 & 0.88 & 0.87 & 0.88 & 0.85 & 0.98 & 0.91 \\
\hline & $\pm \mathrm{SE}$ & 0.05 & 0.08 & 0.04 & 0.01 & 0.04 & 0.02 & 0.07 & 0.05 \\
\hline & $\mathbf{A}$ & & ** & & & & & & \\
\hline & B & & & ** & ** & ** & $\star \star$ & ** & ** \\
\hline
\end{tabular}


Table (6 - B ) : Serum creatinine level in control, diabitic and plant extracts treated rats after2 weeks of recovery period.

\begin{tabular}{|c|c|c|c|c|c|c|c|c|c|}
\hline & \multicolumn{8}{|c|}{ Recovery period ( 2 weeks ) } \\
\hline & & Control & Diabetic & Mixture & $\begin{array}{c}\text { Nigella } \\
\text { sativa }\end{array}$ & $\begin{array}{l}\text { Aloe } \\
\text { vera }\end{array}$ & $\begin{array}{c}\text { Ferulea } \\
\text { assa- } \\
\text { foetida }\end{array}$ & $\begin{array}{c}\text { Boswellia } \\
\text { carterii } \\
\text { Birdw }\end{array}$ & $\begin{array}{c}\text { Commi- } \\
\text { phora } \\
\text { myrrha }\end{array}$ \\
\hline \multirow{4}{*}{ 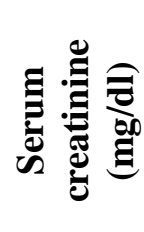 } & Mean & 0.94 & 1.41 & 0.97 & 0.92 & 0.93 & 0.87 & 1.09 & 0.88 \\
\hline & $\pm \mathbf{S E}$ & 0.06 & 0.08 & 0.09 & 0.03 & 0.06 & 0.02 & 0.04 & 0.04 \\
\hline & $\mathbf{A}$ & & $\star \star$ & & & & & & \\
\hline & B & & & $\star * *$ & $\star \star *$ & $\star \star *$ & $\star \star *$ & $\star *$ & $\star *$ \\
\hline
\end{tabular}

A -in comparison with control group .

$\mathrm{B}$-in comparison with diabetic group .

$\mathrm{p}<0.05$.

$* * \mathrm{p}<0.01$.

Table ( 7 - A ) :Total protein concentration in control, diabetic and plant extract treated rats after 4 weeks of treatment .

\begin{tabular}{|c|c|c|c|c|c|c|c|c|c|}
\hline & \multicolumn{8}{|c|}{ Treated period ( 4 weeks ) } \\
\hline & & Control & Diabetic & Mixture & $\begin{array}{l}\text { Nigella } \\
\text { sativa }\end{array}$ & $\begin{array}{l}\text { Aloe } \\
\text { vera }\end{array}$ & $\begin{array}{c}\text { Ferula } \\
\text { assa- } \\
\text { foetida }\end{array}$ & $\begin{array}{c}\text { Boswellia } \\
\text { carterii } \\
\text { Birdw }\end{array}$ & $\begin{array}{c}\text { Commi- } \\
\text { phora } \\
\text { myrrha }\end{array}$ \\
\hline \multirow{4}{*}{ 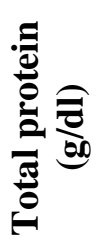 } & Mean & 7.82 & 5.71 & 7.88 & 7.55 & 7.59 & 8.00 & 7.89 & 7.99 \\
\hline & $\pm \mathrm{SE}$ & 0.14 & 0.20 & 0.14 & 0.22 & 0.34 & 0.15 & 0.23 & 0.08 \\
\hline & A & & $\star *$ & & & & & & \\
\hline & B & & & $\star *$ & ** & ** & ** & $\star *$ & $\star *$ \\
\hline
\end{tabular}

Table(7 - B ) :-Total protein concentration in control, diabetic and plant extract treated rats after 2 weeks of recovery period .

\begin{tabular}{|c|c|c|c|c|c|c|c|c|c|}
\hline & \multicolumn{8}{|c|}{ Recovery period ( 2 weeks ) } \\
\hline & & Control & Diabetic & Mixture & $\begin{array}{c}\text { Nigella } \\
\text { sativa }\end{array}$ & $\begin{array}{l}\text { Aloe } \\
\text { vera }\end{array}$ & $\begin{array}{c}\text { Ferula } \\
\text { assa- } \\
\text { foetida }\end{array}$ & $\begin{array}{c}\text { Boswellia } \\
\text { carterii } \\
\text { Birdw }\end{array}$ & $\begin{array}{c}\text { Commi- } \\
\text { phora } \\
\text { myrrha }\end{array}$ \\
\hline \multirow{4}{*}{ مَ } & Mean & 7.97 & 5.62 & 7.98 & 7.44 & 8.05 & 7.80 & 8.02 & 7.85 \\
\hline & $\pm \mathbf{S E}$ & 0.09 & 0.19 & 0.17 & 0.38 & 0.19 & 0.21 & 0.41 & 0.20 \\
\hline & $\mathbf{A}$ & & ** & & & & & & \\
\hline & B & & & ** & ** & ** & ** & ** & ** \\
\hline
\end{tabular}

A -in comparison with control group .

B-in comparison with diabetic group .

$\mathrm{p}<0.05$.

$* * \mathrm{p}<0.01$. 
Table ( 8 - A ) :Albumin concentration in control, diabetic and plant extracts treated rats after 4 weeks of trated period.

\begin{tabular}{|c|c|c|c|c|c|c|c|c|c|}
\hline & \multicolumn{8}{|c|}{ Treated period ( 4 weeks ) } \\
\hline & & Control & Diabetic & Mixture & $\begin{array}{c}\text { Nigella } \\
\text { sativa }\end{array}$ & $\begin{array}{l}\text { Aloe } \\
\text { vera }\end{array}$ & $\begin{array}{c}\text { Ferula } \\
\text { assa- } \\
\text { foetida }\end{array}$ & $\begin{array}{c}\text { Boswellia } \\
\text { carterii } \\
\text { Birdw }\end{array}$ & $\begin{array}{c}\text { Commi- } \\
\text { phora } \\
\text { myrrha }\end{array}$ \\
\hline \multirow{4}{*}{ 音 } & Mean & 4.36 & 3.25 & 4.36 & 4.18 & 3.98 & 3.98 & 3.97 & 4.10 \\
\hline & $\pm \mathrm{SE}$ & 0.16 & 0.13 & 0.17 & 0.11 & 0.15 & 0.11 & 0.16 & 0.09 \\
\hline & $\mathbf{A}$ & & ** & & & & & & \\
\hline & B & & & ** & ** & $\star \star$ & ** & ** & $\star \star$ \\
\hline
\end{tabular}

Table ( 8 - B ) : Albumin concentration in control, diabetic and plant extracts treated rats after 2 weeks of recovery period .

\begin{tabular}{|c|c|c|c|c|c|c|c|c|c|}
\hline & \multicolumn{8}{|c|}{ Recovery period ( 2 weeks ) } \\
\hline & & Control & Diabetic & Mixture & $\begin{array}{c}\text { Nigella } \\
\text { sativa }\end{array}$ & $\begin{array}{l}\text { Aloe } \\
\text { vera }\end{array}$ & $\begin{array}{c}\text { Ferula } \\
\text { assa- } \\
\text { foetida }\end{array}$ & $\begin{array}{c}\text { Boswellia } \\
\text { carterii } \\
\text { Birdw }\end{array}$ & $\begin{array}{c}\text { Commi- } \\
\text { phora } \\
\text { myrrha }\end{array}$ \\
\hline \multirow{4}{*}{ 彰 } & Mean & 4.18 & 3.36 & 4.16 & 4.20 & 4.40 & 4.14 & 4.14 & 4.15 \\
\hline & $\pm \mathrm{SE}$ & 0.14 & 0.16 & 0.12 & 0.14 & 0.13 & 0.20 & 0.15 & 0.18 \\
\hline & $\mathbf{A}$ & & ** & & & & & & \\
\hline & B & & & $\star *$ & ** & ** & ** & ** & ** \\
\hline
\end{tabular}

A -in compaison with control group .

$\mathrm{B}$ - in comparison with diabetic group .

$\mathrm{p}<0.05$.

$* * \mathrm{p}<0.01$.

Table ( 9 - A ) :-Globulin concentration in control, diabetic and plant extracts treated rats after 4 weeks of treatment .

\begin{tabular}{|c|c|c|c|c|c|c|c|c|c|}
\hline & \multicolumn{8}{|c|}{ Treated period ( 4 weeks ) } \\
\hline & & Control & Diabetic & Mixture & $\begin{array}{c}\text { Nigella } \\
\text { sativa }\end{array}$ & $\begin{array}{l}\text { Aloe } \\
\text { vera }\end{array}$ & $\begin{array}{c}\text { Ferula } \\
\text { assa- } \\
\text { foetida }\end{array}$ & $\begin{array}{c}\text { Boswellia } \\
\text { carterii } \\
\text { Birdw }\end{array}$ & $\begin{array}{c}\text { Commi- } \\
\text { phora } \\
\text { myrrha }\end{array}$ \\
\hline \multirow{4}{*}{ 章 } & Mean & 3.46 & 2.46 & 3.52 & 3.37 & 3.61 & 4.02 & 3.21 & 3.89 \\
\hline & $\pm \mathrm{SE}$ & 0.16 & 0.12 & 0.14 & 0.11 & 0.17 & 0.14 & 0.12 & 0.18 \\
\hline & $\mathbf{A}$ & & ** & & & & & & \\
\hline & B & & & ** & ** & ** & ** & ** & ** \\
\hline
\end{tabular}


Tabel (9 - B ) :-Globulin concentration in control, diabetic and plant extracts treated rats after 2 weeks of recovery period .

\begin{tabular}{|c|c|c|c|c|c|c|c|c|c|}
\hline & \multicolumn{8}{|c|}{ Recovery period ( 2 weeks ) } \\
\hline & & Control & Diabetic & Mixture & $\begin{array}{c}\text { Nigella } \\
\text { sativa }\end{array}$ & $\begin{array}{l}\text { Aloe } \\
\text { vera }\end{array}$ & $\begin{array}{l}\text { Ferula } \\
\text { assa- } \\
\text { foetida }\end{array}$ & $\begin{array}{c}\text { Boswellia } \\
\text { carterii } \\
\text { Birdw }\end{array}$ & $\begin{array}{c}\text { Commi- } \\
\text { phora } \\
\text { myrrha }\end{array}$ \\
\hline \multirow{4}{*}{ 章 } & Mean & 3.79 & 2.26 & 3.82 & 3.23 & 3.66 & 3.66 & 3.88 & 3.70 \\
\hline & $\pm \mathbf{S E}$ & 0.21 & 0.13 & 0.25 & 0.20 & 0.21 & 0.28 & 0.37 & 0.25 \\
\hline & $\mathbf{A}$ & & ** & & & & & & \\
\hline & B & & & ** & ** & $\star \star$ & $\star \star$ & $\star \star$ & $\star \star$ \\
\hline
\end{tabular}

A -in comparison with control group

$\mathrm{B}$ - in comparison with diabetic group .

$\mathrm{p}<0.05$.

$* * \mathrm{p}<0.01$.

Table (10 - A ) : Albumin / Globulin ratio ( A / G ratio ) in control, diabetic and plant extracts treated rats after 4 weeks of treatment.

\begin{tabular}{|c|c|c|c|c|c|c|c|c|c|}
\hline & \multicolumn{8}{|c|}{ Treated period ( 4 weeks ) } \\
\hline & & Control & Diabetic & & $\begin{array}{l}\text { Nigella } \\
\text { sativa }\end{array}$ & $\begin{array}{l}\text { Aloe } \\
\text { vera }\end{array}$ & $\begin{array}{c}\text { Ferula } \\
\text { assa- } \\
\text { foetida }\end{array}$ & $\begin{array}{c}\text { Boswellia } \\
\text { carterii } \\
\text { Birdw }\end{array}$ & $\begin{array}{c}\text { Commi- } \\
\text { phora } \\
\text { myrrha }\end{array}$ \\
\hline \multirow{4}{*}{ 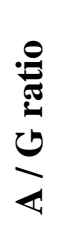 } & Mean & 1.26 & 1.32 & 1.25 & 1.25 & 1.10 & 0.99 & 1.24 & 1.06 \\
\hline & $\pm \mathrm{SE}$ & 0.17 & 0.19 & 0.19 & 0.16 & 0.15 & 0.13 & 0.14 & 0.13 \\
\hline & $\mathbf{A}$ & & - & - & - & - & - & - & - \\
\hline & B & & & - & - & - & - & - & - \\
\hline
\end{tabular}

Table ( 10 - B ) : Albumin / Globulin ratio ( A / G ratio ) control, diabetic and plant extracts treated rats after 2 weeks of recovery period .

\begin{tabular}{|c|c|c|c|c|c|c|c|c|c|}
\hline & & \multicolumn{8}{|c|}{ Recovery period ( 2 weeks ) } \\
\hline & & Control & Diabetic & Mixture & $\begin{array}{l}\text { Nigella } \\
\text { sativa }\end{array}$ & $\begin{array}{l}\text { Aloe } \\
\text { vera }\end{array}$ & $\begin{array}{c}\text { Ferula } \\
\text { assa- } \\
\text { foetida }\end{array}$ & $\begin{array}{c}\text { Boswellia } \\
\text { carterii } \\
\text { Birdw }\end{array}$ & $\begin{array}{c}\text { Commi- } \\
\text { phora } \\
\text { myrrha }\end{array}$ \\
\hline \multirow{4}{*}{ 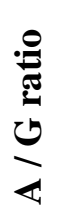 } & Mean & 1.49 & 1.09 & 1.09 & 1.31 & 1.20 & 1.13 & 1.07 & 1.12 \\
\hline & $\pm \mathrm{SE}$ & 0.15 & 0.12 & 0.12 & 0.14 & 0.12 & 0.13 & 0.19 & 0.15 \\
\hline & $\mathbf{A}$ & & - & - & - & - & - & - & - \\
\hline & B & & & - & - & - & - & - & - \\
\hline
\end{tabular}

A - in compaison with control group .

$\mathrm{B}$ - in comparison with diabetic group .

$\mathrm{p}<0.05$.

$* * p<0.01$. 


\section{Discussion}

A significant elevation in LDH activity was detected in heart diseases (Lanter, 1975). In the present work, there was a highly significant increase in the activity of LDH in alloxan diabetic rats. This finding was in agreement with that of Yadav et al. (1997), who stated that increased level of blood glucose in diabetes produces superoxide anions and hydroxyl radicals in the presence of transition metal ions which cause oxidative damage to cell membrane in alloxan diabetic rats. Therefore, it is conceivable to assume that these plants may exert a protective role against the destructive effect of alloxan .

The role of the liver in the metabolism is detoxification and disposition of foreign substances (Mitchell and Jollow, 1975 and Boorman et al., 1990). Transaminases (AST\&ALT), alkaline phosphatase (ALP) and total bilirubin showed an intimate relationship to the cell damage and necrosis and/or increased the permeability of the cell membrane (Boorman et al., 1990). The observed increase in the activity of serum AST, ALT and ALP in the present work may be attributed to the excessive release of such enzymes from the damaged liver cells into the blood circulation. Where, there is an inverse relationship between the liver activity and the level of enzymes in serum (Awadallah and El-Dessouky, 1977).

Our results revealed that, alloxan injection induced maked increase in serum AST and ALT levels. These observation were recorded by many investigators (Helal, 2000 and Youssef and Osman, 2002). The higher levels of these enzymes may be consistent with their greater need for gluconeogenesis substrates or may reflect damage of the hepatic cells due to hepatotoxic effect of alloxan. Rawi et al. (1996) concluded that the elevation in AST and ALT levels might be due to the destruction of the hepatic cells as a result of toxemia.

As regards alkaline phosphatase activity, our results revealed a highly significant increase in diabetic rats at the two tested periods. The elevated activities seen in the present study are parallel with the finding of Abdel-Moneim et al. (2002) who noticed a significant increase in ALP activity in diabetic rats. This elevation appeared to be related to the elevated small intestinal ALP (Chua and Shargo, 1978).

Concerning, serum total bilirubin our data showed a highly significant increase in diabetic rats when compared with control group. This may be due to the damage of hepatic cells or increased destruction of red blood cells with rapid release of bilirubin into the blood (Guyton and Hall, 2000).

While, the group treated with Ferula assafoetida showed a highly significant decrease in serum AST, ALT, ALP and total bilirubin levels when compared with diabetic group but showed a significant increase when compared with control group. This may be attributed to its wild direct effect on the liver cells and the antihepatosis effect (Duke, 2002) .

Otherwise, our results revealed that the oral administration of the water extracts of mixture, Nigella sativa, Aloe vera, Boswellia carterii Birdw and Commiphora myrrha significantly decreased serum AST, ALT, ALP and total bilirubin levels of alloxan diabetic rats. These effects might be attributed to the effect of the tested plants on liver. In this respect, William et al. (1987) reported that certain drugs or their metabolites were able to protect against a direct injury of the liver cells and a doserelated hepatotoxic reaction. Also, this decrease may be ascribed to the improved liver functions with return of glucogneogenesis towards their normal levels. The amelioration of liver functions as shown in the current study, may be ascribed to the insulinogenic effect of these agents as claimed by Hough et al. (1981) who indicated that enzyme activity was completely normalized following insulin administration.

The significant increase of serum urea level may be related to the impairment of renal functions following congestive heart failure. Varley (1976) decided that the blood urea could be increased in all forms 
of kidney diseases such as hydro ephrosis congenital cystic kidney, renal tuberculosis, conditions in which deposition of calcium occurs as hypervitaminosis D. Serum creatinine often raises in type 2 diabetes due to renal arterial diseases and/or cardiac failure rather than to diabetic nephropathy (Guyton and Hall, 2000).

In the present investigation, a significant rise in serum urea and creatinine was observed in alloxan treated rats. This may result from failure of the body to excrete the metabolic end products of proteins (Guyton and Hall, 2000). Where, proteins metabolic rate increased in diabetic as a results of gluconeogenesis increasing rate. Youssef and Osman (2002) reported that alloxan diabetic rats are characterized by considerable tissue damage in kidney that may be indicated by increasing the levels of blood urea and creatinine.

Otherwise, the improvement of serum urea and creatinine levels in rats treated with five tested plants and their mixture may be reflected their effect on improved kidney function and stop the destructive effect of alloxan which in turn may decrease the excessive loss of albumin in urine of diabetic rats.

Alloxan and STZ diabetic animals have a negative nitrogen balance related to enhanced proteolysis in muscles and other tissues and to reduce protein synthesis (Shafrir, 2003).

It was apparent from the results of the present study that serum total proteins, albumin and globulin concentrations showed a significant decrease in alloxan diabetic rats while $\mathrm{A} / \mathrm{G}$ ratio showed nonsignificant change when compared with those of nondiabetic ones. In agreement with these results Helal (2000) and AbdelMoneim et al. (2002) found marked decrease in serum total proteins and albumin in diabetic animals. This decrease in total serum protein content of diabetic rats may be due to the decreased amino acids uptake (Garber, 1980), greatly decreased concentration of a variety of essential amino acids (Brosnan et al., 1984), increased conversion rate of glycogenic amino acids to $\mathrm{CO}_{2}$ and $\mathrm{H}_{2} \mathrm{O}$ (Mortimore and Mandon, 1970), reduction in protein synthesis which in turn may be due to a decrease in the amount and availability of mRNA (Peavy et al., 1985), a loss of translation factor (Wool et al., 1986) and a reduction in ribosomal protein synthesis as a result of insulin deficiency (Jefferson et al., 1983). Wanke and Wong (1991) attributed the decrease of inhibitors of albumin in the experimental diabetes to the presence of albumin promoter activity in liver. Shafrir (2003) attributed the decrease of total proteins and albumin in alloxan diabetic rats to enhanced proteolysis in tissues which affected both reducing production of growth factors and increasing growth factor binding protein by a rapid mechanism and a slowor, long-losting activations of a myofbrillar protease.

Otherwise in our results the treatment of diabetic rats with the water plant extracts and their mixture produced a significant increase in serum total proteins, albumin and globulin concentration while $\mathrm{A} / \mathrm{G}$ ratio showed nonsignificant change when compared with diabetic group throughout the experiment. This increase in serum total proteins and albumin concentration is in harmony with the significant increase of serum insulin level with treatment. Flaim et al. (1985) showed that the decrease of serum total proteins and albumin in diabetic animals was restored to control rats by insulin treatment . Insulin injection accelerates amino acids transport through uptake of amino acids by cells (Werner, 1983) and augmenting incorpor-ation of certain amino acids into proteins (Granner, 1988). Mayer and Shafrir (1984), Pepato et al. (1996) and Aquilani (2004) reported that the inhibition of proteolysis and enhanced protein synthesis is only gradually abolished by insulin.

In the present investigation, it was found that the water extracts of each plant and their mixture not only have no danger effects on liver and kidney functions, but also protects them from the hazard effects of alloxan. So, it is well recommended to use them in treatment of diabetes. Also, further investigation must be done to illustrate this toxicity and their effects on other parameters. 


\section{References}

1. Abdel-Moneim, A.; Al-Zayat, E. and Mahmoud, S. (2002): Effect of some antioxidants on streptozotocin diabetic rats Comparative physiology. J. Egypt. Ger. Soc. Zool. 38(A): 213-245.

2. Aquilani, R. (2004): Oral amino acid admi-nistration in patients with diabetes mellitus: Supplementation or metabolic therapy?. Am. J. Cardiol. 93 (Suppl.): 21- 22.

3. Awadallah, R. and El-dessouky, E. A. (1977): Serum enzyme changes in experim-ental diabetes before and after treatment with some hypoglycemic drugs. J. Ernahrun-gswlss; 16:235-240.

4. Boorman, G.A.; Eustis, S.L.; Elwell, M.R. and Leiniger, J. R. (1990): Pathology of the fisher rat. Reference and Atlas. Academic press. Inc. Harcourt Brace Jovanoich. Publishers: San Diego, New York, Boston, London, Sydney, Tokyo and Toronto, Pp.: 150.

5. Brosnan, J.T.; Man, K.C.; Hall, $H$.E.; Clobourne, S.A. and Brosnan, M.E. (1984): Interorgan metabolism of amino acids in streptozotocin-diabetic rat. Am. J. Physiol., 244: 151- 158.

6. Chua, B. and Shargo, E. (1978): Effects of experimental diabetes and food intake on rat intestine and serum alkaline phosphatase. J. Nutrition, 108:196-202.

7. Doumas, B. T. (1975): Colourimetric determination of total protein in serum or plasma. Clin. Chem., 21(8): 1159-1166.

8. Duke, J.A. (2002): Hand book of medicinal Herbs. $2^{\text {nd }}$ ed. New Interlitho, Millan , Italy, Pp.: 515519.

9. Flaim, K.E.; Hutson, S.M.; Lloyd, C.E.; Taylor,J.M.; Shiman, R. and Jefferson L.S. (1985): Direct effect of insulin on albumin gene expression in primary cultures of rat hepatocytes. Am. J. Physiol., 249:447-453.

10. Garber, A.J. (1980): The impact of streptozotocin-induced diabetes mellitus on cyclic nucleotide regulation of skeletal muscle amino acid metabolism in the rat. J. Clin. Invest., 65: 478-487.

11. Granner, K.D. (1988): Hormones of pancr-eas. In: Harpers Biochemistry. Urray, R.K.; Granner, D.K.; Mayes, P.A. and Rodwell, V.W. editors, $20^{\text {th }}$ ed. Lange Medical Publication, California, Pp. 547-563.

12. Guyton, A.C. and Hall, J.E. (2000): Text book of medical physiology. Endocrinology and reproduction. Insulin, Glucagon and Diabetes Mellitus. $10^{\text {th }}$ ed. W.B. Sounders Company, U.S.A.

13. Helal, E.G.E. (2000): Effectiveness of a herbal mixture with treatment of non-insulin dependent diabetes mellitus. AlAzhar Bull. Sc., 1: 201-324.

14. Hough, S.; Avioli, L.V.; Teitebrum, S.L. and Fallon, M. D. (1981): Alkaline phosph-atase activity in chronic streptozotocininduced insulin deficiency in the rat: Effect of insulin replacement metabolism, 30(12): 1190-1194.

15. Jefferson, L.S.; Warren, S.L.; Peavy, D.E.; Miller, J.B.; Appel, M.C. and Taylor, T.M. (1983): Diabetes induced alterations in liver protein synthesis: Changes in the relative abundance of mRNA for albumin and other plasma proteins. J. Biol. Chem., 258(2): 1369-1375.

16. John, D. (1982): Clinical Laboratory Methods. C.V. Mosby Co., USA, $9^{\text {th }}$ Ed. P. 580-581 .

17. Kachmar, J.F. and Moss, D.w. (1976): In Fundamentals of Clinical chemistry, $2^{\text {nd }}$ ed. NW Tietz, Editor. WB Sounders, Philadelphia, P: 652 .

18. Lanter, A.B. (1975): Clinical biochemistry. $7^{\text {th }}$ ed., Saunder, W.B. Company, Philade-lphia, London, Toronto.

19. Marles, R.T. and Farnsworth, N.R. (1995): Antidiabetic plants and their active constituents. Phytomedicine, 2: 137-189.

20. Mayer, M. and Shafrir, E. (1984): Glucocarticoid and insulin mediated regul-ation of skeletal muscle protein catabolism. In: Shafrir, E. and Renold, A. E., eds. Lessons from animal diabetes. Libbey:P 235. 
21. Mitchell, J.R. and Jollow, O.J. (1975): Metabolic activation of drugs to toxic substances. Gastroenterology, 68:392.

22. Mortimore, G.E. and Mandon, C.E. (1970): Inhibition of insulin of valine turno-ver in liver. J. Biol. Chem., 245: 2375-2383.

23. Okamoto, H. (1984): Molecular basis of experimental diabetes: Degeneration, oncog-enesis and regene-rotion of pancreatic $\beta$-cells of islets of Langerhans. Bio. Essays, 2: 15-21.

24. Patton, C. J. and Crouh, S.R. (1977): A colorimetric method for the determination of blood urea concentration. J. Anal. Chem., 49 : 464-469.

25. Peavy，D.E.; Taylor，J.M. and Jefferson, L.S. (1985): Time course of changes in albumin synthesis and mRNA in diabetic rats. Am. J. physiol., 248: 656-663.

26. Pepato，M.T.; Migliorini, R.H. and Goldberg, A.L. (1996): Role of different proteolytic pathways in degradation of muscle protein from strepto-zotocin-diabetic rats. Am. J. Physiol., 34: 340.

27. Rakieten, N.; Rakieten, M. L. and Nadkarni, M. V. (1963): Studies on the diabetogenic actions of streptozotocin. Cancer. Chemother., 29: 91-98.

28. Rawi, S.M.; Abdel- Moneim, A. and Ahmed, O.M. (1996): Studies on the effect of garlic oil and glibenclamide on alloxan-diabetic rats. 1- Hypoglycemic and histopatho-logical effect. J. Union Arab Biol., 6(A): 121-142.

29. Reitman, S. and Frankel, S. (1975): A colorimetric method for the determination of serum glutamic oxaloacetic and glutamic Pyruvic transaminases. Am. J. Clin. Path., 28: 56.

30. Shafrir, E. (2003): Diabetes in animals: Contribution to the under standing of diabetes by study of its etiopathology in animal models. Bioennial review. Smith-Gordon, Pp.: 231-235.

31. Sokal, R. R. and Rahif, F. J. (1981): The principles and practical of statistic in Biological Research. $2^{\text {nd }}$ ed. Free man, W.H. company, San Frrancisco.

32. Tietz, N.W. (1986): Text book of clinical chemistry. W.B. Saunders Co., London, Philadelphia, Pp.: 1389-1390.

33. Varley, H. (1976): Practical clinical biochemistry . Text book, Indian edition, Publisher (India), PVT Ltd. Sardarjag, Enclave, New Delhi.

34. Wanke, L.E. and Wong, N.C. (1991): Diabetes mellitus decreases the activity of the albumin promoter in vitro. J. Biol. Chem., 266(10): 6068-6072.

35. Webster, D. (1977): Albumin standards and measurements of serum albumin with bromocreasol green. Clin. Chem., $23: 663$.

36. Werner, R. (1983): Essentials of modern biochemistry. ${ }^{\text {st }}$ ed. Jones and Bart Wett publishers. Boston Portala Valley, Pp.: 368-369.

37. William, E.; Balisteri, M. B. and Leslie, M. (1987): Fundamentals of clinical chemistry. $3^{\text {rd }}$ ed., W. B. Saunders Company. Philadelphia, London, Pp.:750-758.

38. Williams, G. and Pickup, J.C. (2000): Hand book of diabetes. $2^{\text {nd }}$ edition. Liverpool, U.K and London UK.

39. Wool, I. G.; Strire-Walt, W. S.; Karrhara, K.; Low, R. B.; Bailey, P. and Oyer, P. (1986): Mode of action of insulin in regulation of protein biosynthesis in muscle. Recent progress in hormone research. New York Academic Press, Pp.: 124-139.

40. Yadav, P.; Sarkar, S. and Rhatnagar, D. (1997): Action of copparis deciduas against alloxaninduced oxidative stress and diabetes in rat tissues. Pharmacol. Res.,36(3): 221-228.

41. Youssef, M.S. and Osman, A.O. (2002): Hypoglycemic effect of Ambrosia maritima and Origanum majorana on alloxan induced diabetic rats. J. Drug Res. Egypt., 24(1-2): 151-153. 


\section{تأثير بعض النباتات الطبية على وظائف الكبل والكلى في الجرذان البيضاء

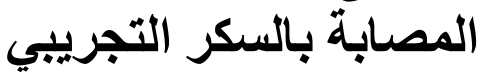

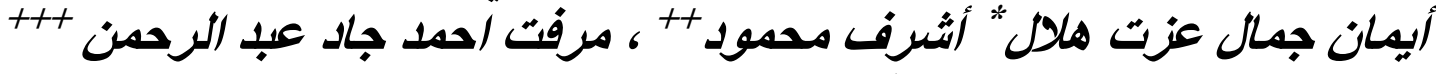

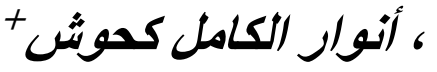 \\ * قسم علم الحيوان ، كلية العلوم - جامعة الأزهر هر (بنات).

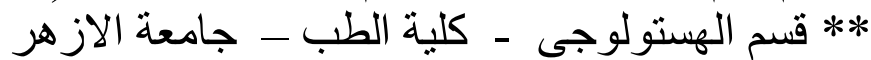

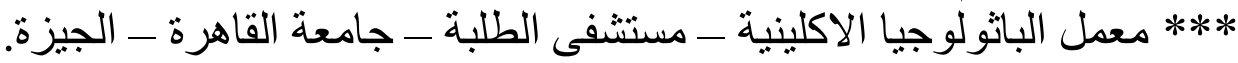

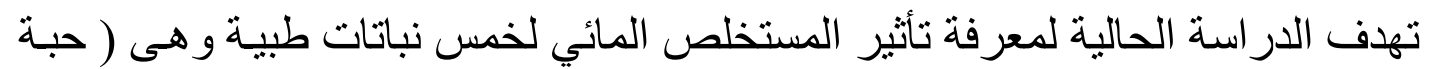

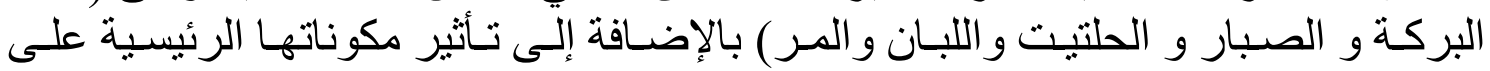

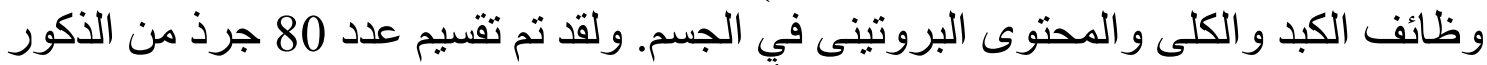

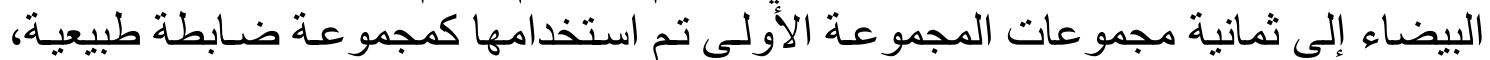

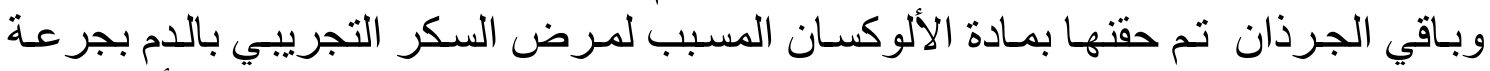

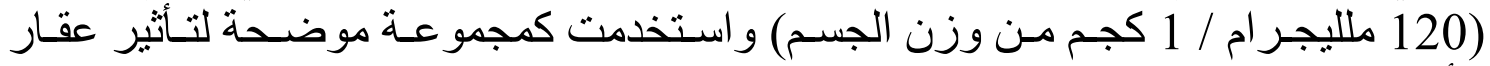

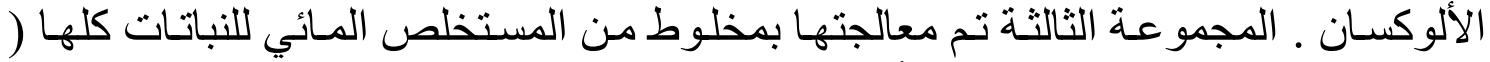

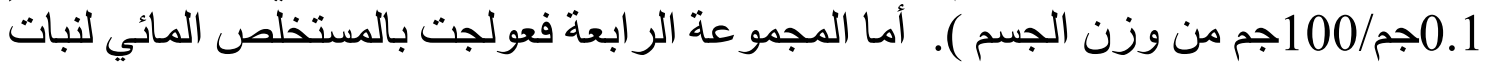

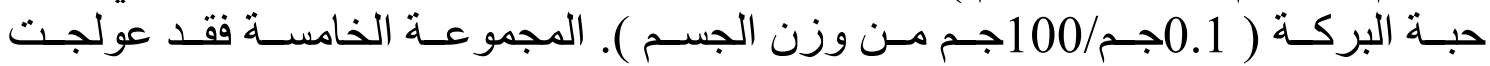

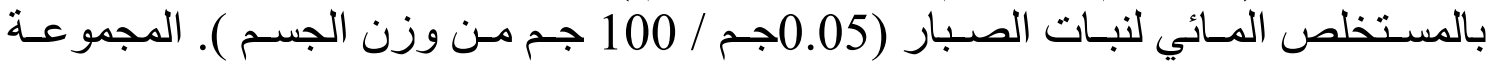

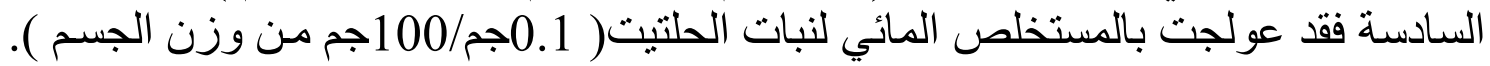

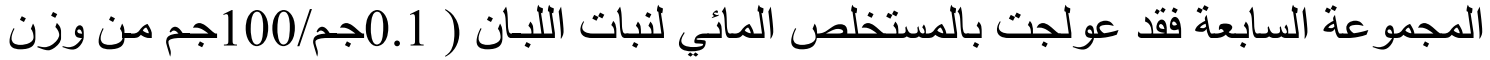

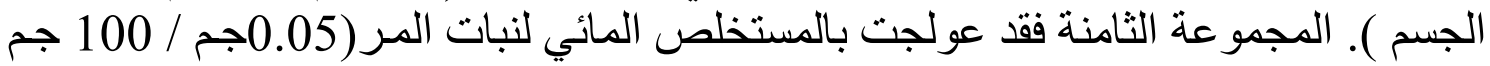

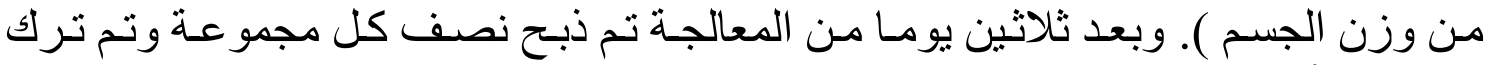
النصف الآخر 15 يو ما كفترة استشفاء

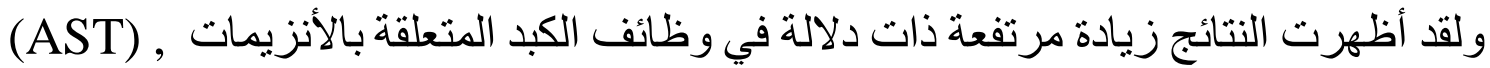

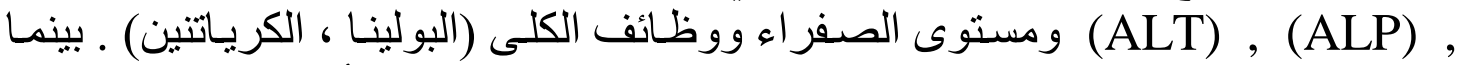

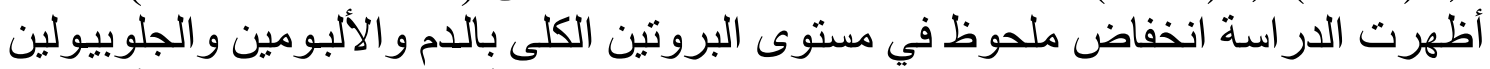

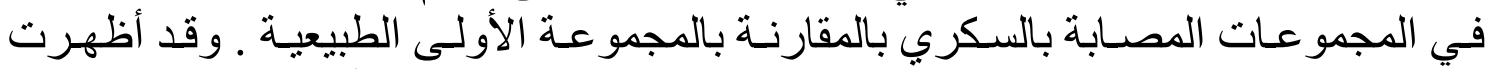

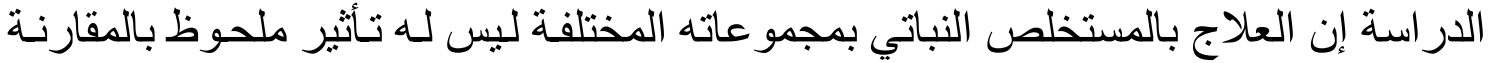
بالمجمو عة الضابطة الطبيعية.

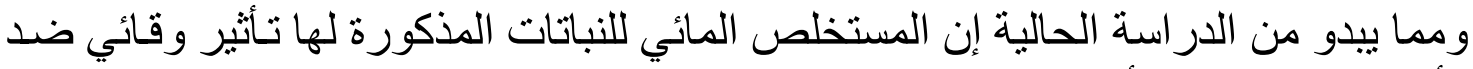
التأثثير السلبي لمادة الألو كسان على وظائف الكبد والكئ الكلى. 